\title{
Perinatal Outcomes in Monozygotic Pregnancies Resulting From Assisted Reproductive Techniques: A Single-Center 6-Year Experience Base on a Large Cohort of Pregnancies
}

\section{Nur Güngör}

Bahcesehir Univercity, Goztepe Medical Park Hospital, IVF Outpatient Clinic

Tuğba Gürbüz ( $\square$ md.tugbagurbuz@gmail.com )

Medistate Hospital Gynecology and Obstetric Clinic, Istanbul

\section{Research Article}

Keywords: Infertility, monozygotic twin, in vitro fertilization, abortus, maternal risk

Posted Date: February 4th, 2021

DOl: https://doi.org/10.21203/rs.3.rs-149411/v1

License: (c) (i) This work is licensed under a Creative Commons Attribution 4.0 International License. Read Full License 


\section{Abstract}

Purpose: Monozygotic twin (MZT) pregnancies increase the risk of maternal and infant mortality and reveal many complications. In the present study, we aimed to share our assisted reproductive techniques (ART) through the analysis of perinatal outcomes in MZT pregnancies.

Materials and Methods: In this retrospective clinical cross-sectional study, 1159 cycles in the In vitro fertilization (IVF) program between October 2014 and December 2019 were retrospectively evaluated and perinatal outcomes and general clinical conditions were analyzed.

Results: 16 cycles with MZT were achieved with IVF, resulting in a MZT incidence of $1.38 \%$. The MZT incidence aged $\leq 35$ and $>35$ years were $0.2 \%$ and $1.1 \%$, respectively. Eight MZT pregnancies resulted in a live birth, while $5 \mathrm{MZT}$ resulted in an abortus. A significant positive correlation was found between the number of trials and the age of female $(r: 0.674 ; p=0.004)$ and male $(r: 0.657 ; p=0.006)$. Cumulus-Oocytes Complexes (COC) (r:0,635; $p=0,008)$, Metaphase II Oocyte (MIIO) (r:0,627; $p=0,009)$, Pronucleus Oocyte (PO) $(r: 0,585 ; p=0,017)$ were correlated with serum AMH levels. Number of MZT was positively correlated with the male age $(r: 0,527 ; p=0,036)$, while negatively correlated with embryo transfer day $(E T d)(r:-0,548$; $p=0,028)$.

Conclusion: The pregnancies that we identified and achieved with IVF had a similar incidence of MZT with the literature, although its risk was more for women $>35$ years. MZT pregnancies can become a problem as the increasing number of IVF applications continue due to its potential risks for both maternal and fetus.

\section{Introduction}

The infertility term, generally known as the failure to perform a reproductive function, is a worldwide health problem in all societies (1). Although infertility treatments vary depending on the source of the problem, age, ovarian difficulty in egg release, alcohol or smoking, intense physical or mental stress are the known causes $(2,3)$. Assisted reproductive techniques (ART), which is the strongest way in treatment today, includes all infertility treatments that ensure the fertilization of eggs and sperm outside the human body $(4,5)$. These are techniques such as pre-implantation genetic screening, embryo cultures, intracytoplasmic sperm injection, fresh or frozen embryo transfer, and in vitro fertilization (IVF) with donor oocytes (6).

ART has been practiced for nearly forty years, and more than a million babies have been born as a result of IVF, and the use and effectiveness of IVF have increased over time (7). Especially frozen embryo transfer and intracytoplasmic sperm injection in this method contribute strongly to these results (8). More than $30 \%$ of pregnancies with ART are multiple pregnancies, and more than half of these newborns are the product of multigestations (9). This causes prematurity and other complications and, consequently, babies born from multiple pregnancies have higher rates of hospitalization and mortality compared to single (10). 
The frequency of anomalies in multiple pregnancies has been reported higher than single pregnancies (11). Except for some problems that can be treated antenatally, non-vital anomalies cause new problems for both obstetricians and parents in multiple pregnancies (12). Although twin births are $2 \%$ of all births, $8 \%$ of perinatal deaths occur in multiple pregnancy cases (13). Mortality is 4 times higher in multiple pregnancies than single pregnancies and perinatal mortality is $19-32 \%(14)$. Specific problems associated with high mortality in multiple pregnancies are common in monochorionic twins (15). Monochorionic placentation is shown as an increase in the frequency of congenital anomalies in monozygotic twin (MZT) pregnancies. Compression-related deformations and lower extremity deformities occurring in the last stages of pregnancy and related to intrauterine posture position are not uncommon in multiple pregnancies (16).

Considering that MZT pregnancies increase the risk of maternal and infant mortality and reveal many complications, sharing rates in ART treatment facilities constitutes importance to ensure a certain standard and to closely monitor the perinatal risks. In the present study, we aimed to share our ART experience through the analysis of perinatal outcomes in MZT pregnancies.

\section{Materials And Methods}

\section{Study Design}

We retrospectively evaluated 1159 cycles that were included in the ICSI program at Department of Obstetrics and Gynecology, Faculty of Medicine, between October 2014 and December 2019. We analyzed the perinatal outcomes and general clinical conditions of all these Monozygotic pregnancies.

This retrospective clinical cross-sectional study was conducted following the Bahcesehir Univercity Ethics Committee approval with the date and number: 17.07.2020-0003. All the experiment protocol for involving humans was in accordance to guidelines of national/international/institutional or Declaration of Helsinki. Informed consent was obtained from all subjects and all the data collected included the basic clinical conditions of the patients, delivery results, type of conception and pregnancy outcomes. Recorded data belonging to the patients were obtained from the retrospective examination result, and was completed by using inpatient treatment charts from our institution. Patient data included in the study were reached with incomplete or inconsistent participants. We evaluated all the cases included in the study in terms of the average age of pregnant women, average gestational week, delivery type, birth weight, presence of EMR, gender, cesarean indications, abortion, fetal problems, fetal reduction, discordance between fetuses, pregnancy anemia, blood transfusion to mother, cerclage, abortus imminens, emesis gravidarum, BMl.

\section{Inclusion \& Exclusion Criteria}

Women were identified through the use of an electronic data. As a result of all clinical and sonographic analyzes, we included MZT pregnancies that reached pregnancy after 16 weeks. All other pregnancy or related conditions were excluded from the present study. In detailed sonographic analysis, pregnancies were accepted as monochorionic according to the presence of placenta and the absence of twin peak 
mark expressed as "lambda sign". Clinical assessment and ultrasound analyzes of the patients were performed by the experienced gynecologist team in obstetric sonography and IVF.

\section{Statistical Analysis}

The SPSS program for Windows (v16.0, SPSS Inc. Illinois,USA) was used to evaluate the study results. Descriptive statistics outputs were given as mean \pm standard deviation for continuous numerical variables or median (minimum-maximum) and percentage for categorical variables. Comparative analysis was done with the Mann-Whitney $U$ test for continuous data and the $\chi 2$ test for categorical data. $P<0.05$ was considered statistically significant for all data assessment.

\section{Results}

In the 1159 cycle, a total of 16 cycles with MZT were achieved with IVF, resulting in a MZT incidence of $1.38 \%$. The MZT incidence aged $\leq 35$ and $>35$ years were $0.2 \%$ and $1.1 \%$, respectively. Given in Table- 1 , the main reason for infertility was male-related factors $(n: 7)$. Other reasons were endometriosis $(n: 3)$, Duchenne muscular dystrophy (n:2), tubal (n:1), and unexplained (n:3). The mean female age was $29.8 \pm$ 3.4 , while it was $33.7 \pm 3.2$ for the male age. The BMI was found as $24.2 \pm 2.3 \mathrm{~kg} / \mathrm{m}^{2}$. The mean value of the trial frequency was $2.1 \pm 2.2$, and the mean AMH level was $2.4 \pm 1.7$.

Eight MZT pregnancies resulted in a live birth. Of those, 2 pregnancies were triplet pregnancy (\%25). Five MZT pregnancies resulted in an abortus. Of those, 2 pregnancies were triplet pregnancy (\%40). Three MZT pregnancies, which were twin pregnancy, resulted in partial abortion. All the patients' details about cycle characteristics were given in Table-2.

A significant positive correlation was found between the number of trials and the age of female (r:0.674; $p=0.004)$ and male $(r: 0.657 ; p=0.006)$. Similarly, Cumulus-Oocytes Complexes $(C O C)(r: 0,635 ; p=0,008)$, Metaphase II Oocyte (MIIO) $(r: 0,627 ; p=0,009)$, Pronucleus Oocyte $(P O)(r: 0,585 ; p=0,017)$ were correlated with serum AMH levels. Number of MZT was positively correlated with the male age $(r: 0,527 ; p$ $=0,036)$, while negatively correlated with embryo transfer day $(E T d)(r:-0,548 ; p=0,028)$.

\section{Discussion}

Today's career planning individuals' approach to marriage at an advanced age and the tendency to delay pregnancy has increased by IVF application worldwide. Although a rare event, previous studies have reported that the incidence of MZT has increased more than four times as a result of IVF procedures, with an increased risk of obstetric complications and poor pregnancy outcomes (17-19). For these reasons, MZT pregnancies can become a problem as the increasing number of IVF continue. In that term, the present study will contribute to the MZT numbers resulting from the application of IVF in our country and the perinatal clinical outcomes of MZT. 
Despite the problematic effects of MZT caused by pregnancies, which we frequently see, it still transfers multiple embryos in many hospitals in our country and around the world to increase the chance of pregnancy (20). The reduction in fertility may be mainly due to oocyte aging rather than poor endometrial receptivity, as older women produce fewer oocytes and have lower implantation rates, suggesting that follicles are less responsive to exogenous hormones and fewer retrieval high-quality oocytes (21). In Sotiroska's study (22), the pregnancy at highest rates were reported in who received ET on 5th day. However, they observed a strong decrease in delivery/pregnancy rates in the higher age group (age $>36$ years) compared to the younger age group. This suggests that older women have a lower chance of conceiving through assisted reproductive technology, despite having low basal FSH levels. In the present study, the number of MZT was positively correlated with the male age, while negatively correlated with embryo transfer day. A significant positive correlation was found between the number of trials and the age of female and male.

In recent studies, various aspects of IVF techniques, parental age, hormone levels, and anatomical problems have previously been discussed concerning MZT incidence. According to Knopman et al. (13), the likelihood of MZT was related to the fact that younger individuals exhibited their possible superior reproductive potential as a result of finding healthier oocytes. Even though this approach is very logical, it is still very uncertain and more data is needed. Embryo derived from younger oocyte is transferred at a blastocyst stage of the advanced levels. This because age factor may not be an independent risk factor for MZT, but rather a representative of blastocyst transfer (23). In the study by Sills et al, the MZT incidence was $1.3 \%$, comparable to reported results, 3 times higher than a natural pregnancy at $0.4 \%(24)$. Vega et al., in the largest study of IVF cycles evaluating multiple pregnancies, dizygotic, and discordant twinning rates, reported the rate of MZT in women < 35 years of age is $1.7 \%(25)$. Osianalis et al. analyzed a large single-institution database and determined that cycles carried a $2.3 \%$ risk of multiple pregnancies (26). In our study with the 1159 cycle, 16 cycles with MZT were achieved with IVF, resulting in a MZT incidence of $1.38 \%$. The MZT incidence aged $\leq 35$ and $>35$ were $0.2 \%$ and $1.1 \%$, respectively.

As a result, high-risk results in terms of maternal and infant health can occur in IVF. Shevell et al. detected an increased abnormal placentation rate in IVF and it was assumed that it may cause these complications during pregnancy. Romundstad et al. found that placenta previa occurs six times more frequently in singleton pregnancies after assisted reproduction. Zhu et al. reported abnormal placental cord placement among women who gave birth after ART and matched controls of spontaneous pregnancies. Considering these studies, it has been suggested that MZT may be the cause of poor maternal and stillbirth outcome events resulting from inadequate or abnormal placental development. In our study, we observed that 8 MZT pregnancies resulted in a live birth. Five MZT pregnancies resulted in an abortus. Three MZT pregnancies, which were twin pregnancy, resulted in partial abortion. These MZT results support increasing risks for maternal health in both our study and literature.

The effect of low AMH levels on oocyte quality is known. However, the possibility that the association between $\mathrm{AMH}$ and the presence of oocyte defects may result from decreased granulose secretion in poor quality oocytes remains an important issue that continues to be investigated. $\mathrm{AMH}$ levels may influence 
the determination of the dominant follicle through the inhibitory effects of AMH during the primary follicle collection process from the primordial pool (27) and regulation of FSH sensitivity in ovarian tissue (28). Borges et al. (29) and Fanchin et al. (30), they examined whether follicular AMH production was positively associated with oocyte and embryo development and demonstrated its significant effect. Ebner et al. showed that AMH levels are directly and strongly associated with oocyte quality (31). While Loh and Maheshwari previously explained that AMH was unable to predict the likelihood of pregnancy (32). La Marca et al. reported that extremely low levels of AMH were associated with the inability to conceive (33). Lamazou et al. found reasonable pregnancy rates following extremely low serum AMH levels (34). Borges et al. showed that the probability of pregnancy and the number of embryos obtained, high-quality embryos, and the number of embryos transferred were positively correlated with the AMH level (29). Similarly, in the present study, we found that cumulus-oocytes complexes, metaphase-Il oocyte, pronucleus oocyte were strongly correlated with serum AMH levels.

The present study had its limitations. All the patients' data about risk factors for MZT in ART-related pregnancies could not be reached in this retrospective study. Another issue is the fact that women who gain pregnancy with IVF and conceive naturally may vary according to demographics that may be associated with the possibility of MZT. Since being a young women is directy connected with the MZT in ART, we cannot exclude the possibility of underestimating the risk. Generally, this limitation could be seen in all clinical observational analyzsis in diffferent medical fields. In the present analysze, however, this should be noted that although some confounders cannot be ruled out, the magnitude of the risk tends to support a causal effect.

\section{Conclusion}

The pregnancies that we identified and achieved with IVF had a similar incidence of MZT with the literature, although its risk was more for women $>35$ years. Strong positive correlation was found between the number of trials and the age of female and male. The number of MZT was positively correlated with the male age, while negatively correlated with embryo transfer day. As a result, MZT pregnancies can become a problem as the increasing number of IVF applications continue due to its potential risks for both maternal and fetus.

\section{Declarations}

Conflict of Interest: The authors have declared no conflict of interest for the present study.

Ethics approval and consent to participate: The study was approved by Ethics Committee of Bahcesehir Univercity with the date and number: 17.07.2020-0003. Informed consent was obtained from all subjects

Consent for publication: Not Applicable (17 July 2020).

Availability of data and materials: All data generated or analysed during the present study are included in this published article.The datasets analysed during the current study available from the corresponding 
author on reasonable request.

Funding: Not applicable.

Authors' contributions: N.D.G. and T.G. planned the study, conducted and wrote the main manuscript text. All authors reviewed the manuscript.

Acknowledgements: Not applicable.

\section{References}

1. Sarac M, Koc I. Prevalence and Risk Factors of Infertility in Turkey: Evidence from Demographic and Health Surveys, 1993-2013. Journal of biosocial science. 2018;50(4):472-90.

2. Eker C, Celik HG, Balci BK, Gunel T. Investigation of human paternal mitochondrial DNA transmission in ART babies whose fathers with male infertility. European journal of obstetrics, gynecology, and reproductive biology. 2019;236:183-92.

3. Inal HA, Kahyaoglu I, Turkkani A, Tuzluoglu D, Yilmaz N. Retrospective comparison of intracytoplasmic sperm injection outcomes of sperm retrieved from a testicular biopsy and freshly ejaculated semen in oligozoospermia. Revista Internacional de andrologia. 2018;16(4):131-6.

4. Aboulghar MM, El-Faissal Y, Kamel A, Mansour R, Serour G, Aboulghar M, et al. The effect of early administration of rectal progesterone in IVF/ICSI twin pregnancies on the preterm birth rate: a randomized trial. BMC pregnancy and childbirth. 2020;20(1):351.

5. Ballesta-Castillejos A, Gomez-Salgado J, Rodriguez-Almagro J, Ortiz-Esquinas I, Hernández-Martínez A. Obstetric and perinatal complications associated with assisted reproductive treatment in Spain. Journal of assisted reproduction and genetics. 2019;36(12):2435-45.

6. Cakiroglu $Y$, Tiras B. Determining diagnostic criteria and cause of recurrent implantation failure. Current opinion in obstetrics \& gynecology. 2020;32(3):198-204.

7. Calik KY, Bulut HK. Assessment of Turkey IVF (In Vitro Fertilization) websites according to the American Society for Reproductive Medicine (ASRM)/Society for Assisted Reproductive Technology (SART) guidelines. JPMA The Journal of the Pakistan Medical Association. 2020;70(3):421-6.

8. Gurbuz AS, Gode F, Ozcimen N. Non-Invasive Isthmocele Treatment: A New Therapeutic Option During Assisted Reproductive Technology Cycles? Journal of clinical medicine research. 2020;12(5):307-14.

9. Jančar N, Mihevc Ponikvar B, Tomšič S, Vrtačnik Bokal E, Korošec S. Is IVF/ICSI [corrected] an Independent Risk Factor for Spontaneous Preterm Birth in Singletons? A Population-Based Cohort Study. BioMed research international. 2018;2018:7124362.

10. Cavoretto P, Candiani M, Giorgione V, Inversetti A, Abu-Saba MM, Tiberio F, et al. Risk of spontaneous preterm birth in singleton pregnancies conceived after IVF/ICSI treatment: a meta-analysis of cohort studies. Ultrasound in obstetrics \& gynecology: the official journal of the International Society of Ultrasound in Obstetrics and Gynecology. 2018;51(1):43-53. 
11. Elias FTS, Weber-Adrian D, Pudwell J, Carter J, Walker M, Gaudet L, et al. Neonatal outcomes in singleton pregnancies conceived by fresh or frozen embryo transfer compared to spontaneous conceptions: a systematic review and meta-analysis. Archives of gynecology and obstetrics. 2020;302(1):31-45.

12. Dobrosavljevic A, Rakic S, Mihajlovic S. Risk of spontaneous preterm labor in pregnancies achieved by in vitro fertilization and complicated with the severe form of ovarian hyperstimulation syndrome: A case-control study. Pakistan journal of medical sciences. 2019;35(4):923-8.

13. Fernandes TR, Carvalho PR, Flosi FB, Baião AE, Junior SC. Perinatal Outcome of Discordant Anomalous Twins: A Single-Center Experience in a Developing Country. Twin research and human genetics: the official journal of the International Society for Twin Studies. 2016;19(4):389-92.

14. Zegers-Hochschild F, Schwarze JE, Crosby J, Musri C, Urbina MT. Assisted reproduction techniques in Latin America: the Latin American Registry, 2014. Reproductive biomedicine online. 2017;35(3):28795.

15. Fineman DC, Baer RJ, Chambers CD, Rajagopal S, Maltepe E, Rinaudo PF, et al. Outcomes of pulmonary vascular disease in infants conceived with non-IVF fertility treatment and assisted reproductive technologies at 1 year of age. Pediatric pulmonology. 2019;54(11):1844-52.

16. Arıoğlu Aydın Ç, Aydın S, Serdaroğlu H. Multifetal gestations with the assisted reproductive technique before the single-embryo transfer legislation: obstetric, neonatal outcomes, and congenital anomalies. The journal of maternal-fetal \& neonatal medicine: the official journal of the European Association of Perinatal Medicine, the Federation of Asia and Oceania Perinatal Societies, the International Society of Perinatal Obstet. 2016;29(15):2475-80.

17. Delrieu D, Himaya E, Phillips S, Kadoch I-J. Monozygotic multiple pregnancies following IVF: a case report series of rare experiences. Reproductive biomedicine online. 2012;25(5):460-5.

18. Franasiak JM, Dondik Y, Molinaro TA, Hong KH, Forman EJ, Werner MD, et al. Blastocyst transfer is not associated with increased rates of monozygotic twins when controlling for embryo cohort quality. Fertility and Sterility. 2015;103(1):95-100.

19. Gee RE, Dickey RP, Xiong X, Clark LS, Pridjian G. Impact of monozygotic twinning on multiple births resulting from in vitro fertilization in the United States, 2006-2010. American Journal of Obstetrics and Gynecology. 2014;210(5):468.e1-.e6.

20. Tummers P. Risk of spontaneous abortion in singleton and twin pregnancies after IVF/ICSI. Human Reproduction. 2003;18(8):1720-3.

21. Chuang C-C, Chen C-D, Chao K-H, Chen S-U, Ho H-N, Yang Yu-S. Age is a better predictor of pregnancy potential than basal follicle-stimulating hormone levels in women undergoing in vitro fertilization. Fertility and Sterility. 2003;79(1):63-8.

22. Sotiroska V, Petanovski Z, Dimitrov G, Hadji-Lega M, Shushleski D, Saltirovski S, et al. The day of embryo transfer affects the delivery rate, birth weights, female-to-male ratio, and monozygotic twin rate. Taiwanese Journal of Obstetrics and Gynecology. 2015;54(6):716-21. 
23. Knopman JM, Krey LC, Oh C, Lee J, McCaffrey C, Noyes N. What makes them split? Identifying risk factors that lead to monozygotic twins after in vitro fertilization. Fertility and Sterility. 2014;102(1):82-9.

24. Sills ES, Moomjy M, Zaninovic N, Veeck LL, McGee M, Palermo GD, et al. Human zona pellucida micromanipulation and monozygotic twinning frequency after IVF. 2000;15(4):890-5.

25. Vega M, Zaghi S, Buyuk E, Jindal S. Not all twins are monozygotic after elective single embryo transfer: analysis of 32,600 elective single embryo transfer cycles as reported to the Society for Assisted Reproductive Technology. Fertility and Sterility. 2018;109(1):118-22.

26. Osianlis T, Rombauts L, Gabbe M, Motteram C, Vollenhoven B. Incidence and zygosity of twin births following transfers using a single fresh or frozen embryo. Human Reproduction. 2014;29(7):1438-43.

27. Durlinger AL, Gruijters MJ, Kramer P, Karels B, Kumar TR, Matzuk MM, et al. Anti-Müllerian hormone attenuates the effects of FSH on follicle development in the mouse ovary. Endocrinology. 2001;142(11):4891-9.

28. Kevenaar ME, Themmen AP, Laven JS, Sonntag B, Fong SL, Uitterlinden AG, et al. Anti-Müllerian hormone and anti-Müllerian hormone type II receptor polymorphisms are associated with follicular phase estradiol levels in normo-ovulatory women. Human reproduction (Oxford, England). 2007;22(6):1547-54.

29. Borges E, Braga DPAF, Setti A, Figueira RdC, laconelli A, Jr. The predictive value of serum concentrations of anti-Müllerian hormone for oocyte quality, fertilization, and implantation. JBRA Assist Reprod. 2017;21(3):176-82.

30. Fanchin R, Mendez Lozano DH, Frydman N, Gougeon A, di Clemente N, Frydman R, et al. AntiMüllerian hormone concentrations in the follicular fluid of the preovulatory follicle are predictive of the implantation potential of the ensuing embryo obtained by in vitro fertilization. The Journal of clinical endocrinology and metabolism. 2007;92(5):1796-802.

31. Ebner T, Sommergruber M, Moser M, Shebl O, Schreier-Lechner E, Tews G. Basal level of antiMüllerian hormone is associated with oocyte quality in stimulated cycles. Human reproduction (Oxford, England). 2006;21(8):2022-6.

32. Loh JS, Maheshwari A. Anti-Mullerian hormone-is it a crystal ball for predicting ovarian aging? Human reproduction (Oxford, England). 2011;26(11):2925-32.

33. La Marca A, Nelson SM, Sighinolfi G, Manno M, Baraldi E, Roli L, et al. Anti-Müllerian hormone-based prediction model for live birth in assisted reproduction. Reproductive biomedicine online. 2011;22(4):341-9.

34. Lamazou F, Genro V, Fuchs F, Grynberg M, Gallot V, Achour-Frydman N, et al. [Serum AMH level is not a predictive value for IVF in the modified natural cycle: analysis of 342 cycles]. Journal de gynecologie, obstetrique et biologie de la reproduction. 2011;40(3):205-10.

\section{Tables}


Table-1. Demographic details of the patients

\begin{tabular}{|llllll|}
\hline Female Age & Male Age & BMI & Trial & AMH & Reason \\
\hline 26 & 30 & 28 & 0 & 3 & Genetic-DMD \\
\hline 36 & 39 & 22 & 8 & 1 & Male-Related \\
\hline 28 & 31 & 23 & 0 & 0,7 & Unexplained Infertility \\
\hline 29 & 34 & 23 & 2 & 1 & Unexplained Infertility \\
\hline 25 & 35 & 24 & 3 & 0,7 & Male-Related \\
\hline 27 & 36 & 24 & 0 & 5 & Male-Related \\
\hline 25 & 27 & 22 & 2 & 2 & Male-Related \\
\hline 31 & 34 & 27 & 4 & 1 & Tubal \\
\hline 27 & 30 & 25 & 0 & 4 & Genetic-DMD \\
\hline 31 & 33 & 22 & 2 & 5 & Endometriosis \\
\hline 31 & 38 & 24 & 3 & 1 & Unexplained Infertility \\
\hline 30 & 32 & 23 & 0 & 1 & Endometriosis \\
\hline 32 & 35 & 26 & 2 & 1 & Male-Related \\
\hline 36 & 38 & 27 & 5 & 5 & Male-Related \\
\hline 30 & 33 & 20 & 0 & 3 & Endometriosis \\
\hline 32 & 34 & 27 & 2 & 4 & Male-Related \\
\hline
\end{tabular}

Abbreviations. DMD: Duchenne Muscular Dystrophy

Table-2. Patients' outcomes with oocyte and trigger details 


\begin{tabular}{|lllllll|}
\hline Trigger Agent & Outcome & COC & MII & PO & ETD & ETF \\
\hline Ovitrelle & Live Birth-Twin & 12 & 10 & 10 & 5 & 1 \\
\hline Ovitrelle & Live Birth-Twin & 8 & 7 & 7 & 5 & 2 \\
\hline Ovitrelle & Live Birth-Twin & 3 & 3 & 3 & 5 & 1 \\
\hline Ovitrelle & Live Birth-Three & 3 & 3 & 3 & 4 & 2 \\
\hline Ovitrelle & Live Birth-Three & 3 & 3 & 3 & 3 & 2 \\
\hline Ovitrelle & 23w Two Baby Ex & 5 & 4 & 3 & 5 & 1 \\
\hline Ovitrelle & Live Birth-One & 8 & 8 & 6 & 5 & 2 \\
\hline Ovitrelle & Live Birth-Twin & 9 & 7 & 6 & 5 & 2 \\
\hline Ovitrelle & Live Birth-Twin & 9 & 8 & 8 & 5 & 1 \\
\hline Lucrin & Live Birth-One & 14 & 13 & 12 & 5 & 2 \\
\hline Dual & 22w Three Abort & 5 & 4 & 4 & 5 & 2 \\
\hline Ovitrelle & 11w Twin Missed & 7 & 6 & 6 & 5 & 1 \\
\hline Lucrin & 15w Abort-Three & 10 & 8 & 8 & 5 & 2 \\
\hline Lucrin & Live Birth-One & 15 & 12 & 11 & 5 & 2 \\
\hline Lucrin & Live Birth & 11 & 9 & 8 & 5 & 1 \\
\hline Lucrin & Spontan Abort & 11 & 8 & 8 & 5 & 2 \\
\hline
\end{tabular}

Abbreviations. COC: Cumulus-Oocytes Complexes, MII: Metaphase II Oocytes, PO: Pronucleus Oocytes, ETD: Embryo Transfer Day, ETF: Frequency of Embryo Transfer 